\title{
Le soutien aux travailleurs autonomes : constats issus de la recherche et réflexions nourries par le terrain
}

\author{
Sylvie Laferté \\ Téluq \\ L'université à distance de l'UQAM
}

\section{Introduction}

Le travail autonome n'est pas nouveau en soi. Avant la révolution industrielle, il était plutôt la norme alors que tous et chacun gagnait sa vie de la terre ou d'un métier exercé à son propre compte. Suite à la révolution industrielle, le travail autonome a été remplacé par le travail salarié comme principale source de revenu personnel. Cependant, cette forme d'emploi, ou d'entrepreneuriat selon notre vision de la chose, a connu une certaine croissance (oserait-on dire un retour aux sources) depuis une trentaine d'années, suivie d'une stabilisation du nombre de travailleurs autonomes autour de 375000 personnes au Québec depuis le début des années 2000 (en-

En tant que tels, ces travailleurs autonomes bénéficient, au démarrage de leur entreprise et si leur projet répond à un certain nombre de critères, de l'aide des organismes de développement économique,
Cette situation découle non pas d'un manque de bonne volonté ou d'un manque d'offres de formation, de services-conseil ou de livres et guides sur la gestion des petites entreprises. Elle provient plutôt de financement, parfois aléatoire, des organismes de développement économique et des choix que leurs gestionnaires ont à faire concernant l'allocation de leurs ressources. De plus, souvent, ces organismes sont évalués, puis financés, à partir du nombre d'entreprises et d'emplois qu'ils ont aidé à créer, ce qui implique très raisonnablement que leurs ressources seront affectées au soutien au démarrage et à la gestion des plus grandes entreprises plutôt qu'au soutien à la gestion des plus petites entreprises n'impliquant que deux ou trois emplois.

Loin de nous l'idée de leur jeter la première pierre. Au contraire, l'objectif que nous poursuivons ici est de présenter notre réflexion sur le soutien aux travailleurs autonomes en exercice; réflexion basée sur plus de 20 ans d'expérience et de recherche auprès des travailleurs autonomes. Mais avant d'aller plus avant dans cette réflexion, débutons par définir le sujet : le travail autonome.

\section{C'est quoi le travail autonome?}

Celle ou celui qui répondra à cette question gagnera le gros lot! En effet, la définition du travail autonome et du travailleur autonome n'est pas évidente. Certains s'entendent pour dire qu'il s'agit d'une personne seule qui possède, dirige et opère une entreprise $\underline{\text { sans }}$ aucune aide rémunérée. D'autres voient le travail autonome comme une microentreprise, c'est-à-dire une personne, seule ou en associa- 
$\underline{\text { tion }}$ avec une ou plusieurs autres, qui possède, dirige et opère une entreprise avec l'aide de quelques employés (on s'entend généralement sur le nombre, soit moins de cinq (5) employés). Au Canada anglais et aux États-Unis, on parle de «selfemployed» que nous pourrions traduire par « autoemployé », mais qu'eux traduisent par travail indépendant, et leur définition du concept ressemble aux deux définitions précédentes. D'autres encore conçoivent le travail autonome comme une forme de travail atypique comme le télétravail, le cumul d'emplois et les contrats à durée déterminée. Même les travailleurs autonomes eux-mêmes ne s'entendent pas sur le sujet. Plusieurs professionnels, à leur propre compte, ne s'identifient pas comme travailleur autonome (avocat, notaire, ingénieur, courtier d'assurance ou en valeur mobilière, etc.). D'autres sont classés comme travailleurs autonomes par les instances gouvernementales mais qui, selon nous, sont plutôt des salariés de l'état (par exemple les médecins et les propriétaires de centres de la petite enfance ou de garderies en milieu familial). Bref, il est difficile de trancher... tout dépend du point de vue de chacun.

Pour les fins de notre présente réflexion, définissons le travail autonome comme le fait d'une personne qui possède, dirige et opère une entreprise de très petite taille (moins de cinq personnes en tout, employés et associés compris) mais qui ne dépend pas d'un seul donneur d'ordres (comme c'est le cas des médecins qui perçoivent la totalité, ou presque, de leur revenu de l'état, même s'ils ont leur propre bureau de consultation). Cette définition ne plaira pas à tous mais elle a l'avantage de situer le travail autonome dans le contexte de l'entreprise privée.

\section{Le soutien aux travailleurs autonomes : constats issus de la recherche}

Avant de s'attarder au soutien aux travailleurs autonomes, penchons-nous quelques instants sur les raisons qui poussent les travailleurs autonomes à demander de l'aide aux organismes de développement économique. Comme toutes les autres entreprises, quelle qu'en soit la taille, les travailleurs autonomes rencontrent des problèmes de gestion et, c'est lorsqu'ils font face à ces problèmes qu'ils demandent généralement de l'aide aux organismes de développement économique. Une revue de la littérature sur le sujet de même que nos propres recherches ${ }^{1}$ indiquent que les problèmes des travailleurs autonomes sont les mêmes, à peu de choses près, que ceux des entreprises de plus grandes tailles, ce qui nous conforte dans notre opinion que le travail autonome est une forme d'entreprise à part entière. Parmi tous les problèmes de gestion recensés, mentionnons les problèmes liés au marketing (vente, mise en marché, publicité, etc.), à la production (qualité, main-d'œuvre, équipement, emplacement, etc.) et à la gestion des ressources humaines (recrutement, formation et rétention). Au-delà de la gestion, d'autres problèmes font aussi surface auprès des propriétaires d'entreprises de toutes tailles; mentionnons le manque de temps pour tout faire, le sentiment d'isolement, l'insécurité financière, et la difficulté de concilier vie familiale et vie professionnelle.

Tableau 1

Pertinence des services et des activités selon le pourcentage de répondants les ayant évalué 4 ou 5 sur 5 , en ordre de pertinence relative

\begin{tabular}{|l|r|r|}
\hline \multicolumn{1}{|c|}{ Énoncés de services et d'activités } & MOY / 5 & \multicolumn{1}{c|}{$\%$} \\
\hline Formation (cours ou autres formats de formation) & 3,56 & 57,6 \\
\hline $\begin{array}{l}\text { Réseautage (regroupement de travailleurs autonomes et / ou propriétaires de petites entreprises dans un } \\
\text { but de support, d'entraide mutuelle) }\end{array}$ & 3,56 & 52,6 \\
\hline $\begin{array}{l}\text { Regroupement de travailleurs autonomes et / ou propriétaires dirigeants de petites entreprises, dans le but } \\
\text { de défendre nos intérêts (lobby) }\end{array}$ & 3,49 & 51,8 \\
\hline $\begin{array}{l}\text { Aide conseil à des aspects particuliers de gestion par des spécialistes (tenue de livres et comptabilité, } \\
\text { choix des bons moyens de publicité, etc.) }\end{array}$ & 3,48 & 54,1 \\
\hline $\begin{array}{l}\text { Maillage de travailleurs autonomes et / ou propriétaires dirigeants de petites entreprises afin de partager } \\
\text { nos expertises et offrir des produits ou des services plus complets }\end{array}$ & 3,44 & 48,6 \\
\hline $\begin{array}{l}\text { Rencontres ponctuelles entre travailleurs autonomes et / ou propriétaires dirigeants de petites entreprises } \\
\text { pour échanger sur nos problèmes et nos succès (par exemple 5 à 7 ou petit déjeuner échange) }\end{array}$ & 3,40 & 47,1 \\
\hline
\end{tabular}




\begin{tabular}{|c|c|c|}
\hline Énoncés de services et d'activités & MOY / 5 & $\%$ \\
\hline Guichet d'information sur les services et les programmes d'aide financière offerts par les organismes & 3,33 & 51,7 \\
\hline Conférences données par des spécialistes & 3,29 & 48,3 \\
\hline $\begin{array}{l}\text { Regroupement de travailleurs autonomes et / ou propriétaires dirigeants de petites entreprises dans le but } \\
\text { d'échanger des services concrets (par exemple : achat, recrutement du personnel) }\end{array}$ & 3,28 & 44,4 \\
\hline Site internet d'information sur les divers aspects de la gestion et des opérations d'une entreprise & 3,20 & 43,9 \\
\hline $\begin{array}{l}\text { Parrainage ou mentorat (accompagnement par une personne d'affaires d'expérience - aspect gestion de } \\
\text { l'entreprise) }\end{array}$ & 3,18 & 45,2 \\
\hline Conférences données par des entrepreneurs (témoignages) & 3,16 & 41,7 \\
\hline $\begin{array}{l}\text { Compagnonnage (accompagnement par une personne d'expérience, du même secteur d'activités - aspect } \\
\text { technique ou de production de l'entreprise) }\end{array}$ & 3,13 & 43,6 \\
\hline $\begin{array}{l}\text { Promotion des sources régionales d'information statistique et d'information statistique sur le marché } \\
\text { (nous les faire connaître) }\end{array}$ & 3,06 & 42,8 \\
\hline $\begin{array}{l}\text { Aide conseil à la gestion quotidienne par des généralistes (prise de décision, planification, évaluation des } \\
\text { résultats, etc.) }\end{array}$ & 3,05 & 37,3 \\
\hline Publication d'une liste ou d'un bottin des nouvelles entreprises dans ma région & 3,00 & 37,3 \\
\hline $\begin{array}{l}\text { Centre d'affaires (locaux privés où les entreprises peuvent se regrouper physiquement, à long terme ou à } \\
\text { l'occasion, et partager des services communs) }\end{array}$ & 2,96 & 40,5 \\
\hline $\begin{array}{l}\text { Expositions ou foires avec des kiosques pour présenter nos entreprises à des clients ou acheteurs poten- } \\
\text { tiels }\end{array}$ & 2,86 & 35,8 \\
\hline $\begin{array}{l}\text { Incubateur ou pépinière (locaux subventionnés en partie où l'entreprise peut passer ses deux premières } \\
\text { années d'existence et profiter de services divers) }\end{array}$ & 2,70 & 35,0 \\
\hline Aide conseil à la production et aux opérations par des spécialistes du secteur d'activités. & 2,69 & 31,5 \\
\hline Identification des produits et des services comme étant d'origine locale ou régionale & 2,62 & 32,3 \\
\hline Campagne d'achat local ou régional & 2,62 & 31,1 \\
\hline $\begin{array}{l}\text { Site internet «chat» (site d'échange avec d'autres gens d'affaires, travailleurs autonomes et /ou proprié- } \\
\text { taires dirigeants de petites entreprises) }\end{array}$ & 2,38 & 20,2 \\
\hline Moyenne de l'échelle & 3,11 & \\
\hline
\end{tabular}

Lors d'une enquête ${ }^{2}$ auprès de 257 travailleurs autonomes québécois (Laferté, 2005), nous leur avons demandé de nous indiquer, sur une échelle de 1 à 5 , la pertinence de 23 services et activités pouvant les aider à résoudre leurs problèmes et qui sont, ou pourraient, être offerts par les organismes de déve- loppement économique. Le tableau 1 présente les résultats obtenus. Nous leur avons aussi demandé d'évaluer leur intérêt envers 10 façons de participer ou de suivre une activité de formation. Les résultats obtenus sont présentés au tableau 2.

\section{Tableau 2 \\ Intérêt pour les formats de formation selon le pourcentage de répondants les ayant évalué 4 ou 5 sur 5 , en ordre d'intérêt relatif}

\begin{tabular}{|l|r|r|}
\hline \multicolumn{1}{|c|}{ Énoncés de formats de formation } & MOY / 5 & \multicolumn{1}{|c|}{$\%$} \\
\hline $\begin{array}{l}\text { Atelier ou séminaire (théorie et pratique, travail en petits groupes ou individuel afin de } \\
\text { mettre en pratique les notions théoriques reçues) }\end{array}$ & 3,28 & 47,1 \\
\hline $\begin{array}{l}\text { Documentation écrite (livres, brochures, cahiers...) } \\
\text { Colloque (sur une ou plusieurs journées, des personnes ressources parlent des aspects pra- } \\
\text { tiques de leur spécialité) }\end{array}$ & 3,18 & 40,1 \\
\hline Encadrement individuel (aide et conseil) à l'extérieur de l'entreprise & 42,1 \\
\hline Encadrement individuel (aide et conseil) sur les lieux mêmes de l'entreprise & 3,13 & 38,9 \\
\hline $\begin{array}{l}\text { Cédérom interactif sur un sujet en particulier, avec ou sans suivi « en ligne » (apprendre à } \\
\text { votre propre rythme à partir de votre ordinateur) }\end{array}$ & 3,09 & 40,5 \\
\hline
\end{tabular}




\section{Énoncés de formats de formation}

Cours en groupe (formule traditionnelle sur un sujet précis, présence physique, horaire régulier, contenu plutôt théorique)

Cours à distance (travailler de chez-soi, à votre propre rythme, à partir de matériel pédagogique écrit ou électronique, contenu plutôt théorique)

Forum d'experts sur internet (vous posez vos questions « en ligne » et un expert vous répond)

Vidéo explicative sur un sujet en particulier

Moyenne générale

MOY / $5 \quad \%$

$2,85 \quad 31,5$

$2,76 \quad 33,8$

$2,70 \quad 30,0$

2,66

25,6

2,99

Après quelques manipulations statistiques dont nous vous épargnons les détails ${ }^{3}$, nous avons constaté que les activités impliquant des rencontres ou des contacts entre les travailleurs autonomes (réseautage, regroupement (lobby), maillage, etc.) étaient jugées comme étant les plus pertinentes, suivies de celles directement axées sur la formation (conférences de spécialistes ou d'entrepreneurs et formation), de celles où les travailleurs autonomes obtiendraient des conseils (parrainage ou mentorat, aide à la gestion par des spécialistes ou des généralistes, incubateur ou pépinière, etc.), de celles nécessitant l'utilisation d'internet (site internet de gestion, guichet d'information et chat), et enfin de celles plutôt axées sur la promotion (campagne d'achat local, expositions ou foires). Nous avons aussi constaté que les formats de formation où les travailleurs autonomes se retrouveraient entre eux (cours en groupe, colloque, atelier ou séminaire) étaient évalués comme étant plus intéressants que les formats de formation où ils travailleraient plutôt seuls (cours à distance, forum d'experts sur internet, vidéo et cédérom interactif).

Dans le contexte de notre définition de travail autonome, nous avons vérifié si la présence ou l'absence d'aide rémunérée avait une influence sur l'évaluation de la pertinence des activités et services, et sur l'intérêt envers les formats de formation. En aucun cas, les tests statistiques que nous avons utilisés n'ont indiqué que cette présence ou cette absence d'employés ait eu une influence significative. En ce qui concerne le nombre de personnes impliquées dans l'entreprise, la seule différence que nous avons trouvée est que les travailleurs autonomes ayant un ou quelques associés évaluent les activités de type regroupement comme étant moins pertinentes que ne le font les travailleurs autonomes sans associés.

\section{Le soutien aux travailleurs autonomes : réflexions nourries par le terrain}

Nous avons discuté de ces résultats avec plusieurs personnes provenant de différents milieux (universitaires, intervenants économiques, et représentants d'organismes de soutien, de regroupement ou de représentation des travailleurs autonomes). Ces résultats, ces discussions et notre expérience antérieure auprès de cette clientèle nous amènent à formuler quelques recommandations aux organismes de développement économique ${ }^{4}$.

Nous avons tous vécu la situation où, dans une activité $\mathrm{X}$ ou $\mathrm{Y}$, la traditionnelle pause de 15 minutes n'a pas été suffisante afin de terminer une conversation, un échange fructueux, avec une personne partageant nos préoccupations. Les travailleurs autonomes, souvent seuls à la tête de leur entreprise, apprécieraient certainement pouvoir échanger avec des pairs, des experts et autres personnes ressources lors d'activités organisées à leur profit par les organismes de développement économique (formation, conférence, témoignage, etc.). En ce sens, notre première recommandation est de prévoir, lors d'activités où sont invités les travailleurs autonomes, des périodes d'au moins une heure pendant lesquelles ils pourront échanger librement ou sur la thématique de l'activité en question.

Malgré le fait que les activités de regroupement, qui permettent des échanges entre les participants, ont été évaluées comme étant les plus pertinentes, les résultats présentés au tableau 1 indiquent que la formation arrive en tête de liste comme type d'activité la plus pertinente. Comme l'atelier ou le séminaire, alliant théorie et pratique, semble être le 
format de formation le plus pertinent (tableau 2), notre deuxième recommandation est que les activités de formation devraient, prioritairement, utiliser cette approche ou une approche permettant aux participants de comprendre (théorie) et d'appliquer (pratique) les sujets traités, tout en permettant des périodes d'échange.

Le principal problème des travailleurs autonomes est le manque de temps pour tout faire (Laferté, 2005), notre troisième recommandation est que les activités de soutien offertes par les organismes de développement économique devraient donc être de courte durée ou à durée flexible; par exemple, une activité de formation d'une durée maximale de trois (3) heures, ce qui permettrait de la réaliser sur un tiers de journée, soit en avant-midi, en après-midi ou en soirée, ou encore sur des périodes d'une heure lors de petits-déjeuners, de lunchs ou de dîners, étalées sur une ou plusieurs semaines, le cas échéant, afin d'approfondir le sujet.

À certains égards, la majorité des activités de soutien présentées au tableau 1 peuvent s'organiser autour de périodes de courte durée ou à durée flexible. Les activités de réseautage, de maillage, les rencontres ponctuelles, les conférences, le parrainage et l'aide conseil, pour n'en mentionner que quelques uns, y sont particulièrement bien adaptées. Quant aux formats de formation les plus intéressants selon les répondants (tableau 2), plusieurs d'entre eux répondent bien aussi à cette flexibilité dans les horaires; mentionnons la documentation écrite, l'encadrement, le cédérom interactif et la formation à distance.

\section{Le principal problème des travailleurs autonomes est le manque de temps pour tout faire}

Nous avons déjà mentionné que les travailleurs autonomes semblent préférer la formation de type atelier ou séminaire, alliant théorie et pratique (tableau 2). Ce type de formation est déjà offert, entre autres par les organismes de développement économique. Cependant, les contraintes inhérentes aux horaires et aux coûts de cette formation nuisent à la participation des travailleurs autonomes. En ce sens, même si le format de formation à distance ne s'est pas positionné dans les premiers choix des répon- dants, il pourrait devenir une solution intéressante à la contrainte de flexibilité des horaires. Notre quatrième recommandation est de procéder à une réflexion en profondeur sur les sujets et la forme de l'enseignement à distance afin de répondre aux besoins particuliers des plus petites entreprises; par exemple, en utilisant les technologies de l'information maintenant disponibles, il serait possible d'inclure des temps d'échange virtuel entre les participants tout en leur fournissant un contenu tout autant théorique que pratique.

Avant de conclure, mentionnons que le problème de manque de temps, qu'il soit dû à une mauvaise organisation $d u$ travail ou non, entraîne une autre conséquence sur l'offre d'activités par les organismes de développement économique; ceux-ci se doivent, et ceci représente notre cinquième recommandation, d'être les porteurs de ces activités. Les travailleurs autonomes n'ont malheureusement pas le loisir d'investir du temps dans l'organisation de telles activités, que ce soit pour eux ou pour leurs pairs. D'ailleurs, selon notre propre expérience auprès d'associations ou regroupements de travailleurs autonomes, à maintes reprises, ces organisations n'ont survécu que le temps de quelques rencontres. Les bénévoles, tous des travailleurs autonomes, ayant quelque peu délaissé leur entreprise au bénéfice de l'organisation d'activités pour leurs pairs, ont rapidement pris conscience du coût des occasions d'affaires ratées durant leur implication dans ces organisations.

\section{Conclusion}

Afin d'investir leurs rares ressources dans des activités de soutien qui pourraient aider les travailleurs autonomes à mieux gérer leur entreprise nous avons formulé 5 recommandations aux organismes de développement économique. Parmi celles-ci et sans amoindrir l'importance des autres, la cinquième nous semble la plus prometteuse.

En résumé, nous recommandons aux organismes de développement économique de soutenir, financièrement et logistiquement, d'abord des activités de types regroupement (réseautage et maillage par exemple), et des activités de formation, notamment sous la forme d'atelier ou de séminaire alliant théorie et pratique et ce, sans négliger les autres formes 
de soutien déjà offertes, et en prévoyant des périodes où les travailleurs autonomes pourraient échanger entre eux sur différentes thématiques. L'application de ces recommandations permettrait aux organismes de développement économique d'investir leurs ressources dans des activités de groupe, répondant aux besoins de plusieurs travailleurs autonomes en même temps. Deux objectifs seraient alors atteints : un soutien mieux ciblé auprès des travailleurs autonomes dans la gestion de leur entreprise et une affectation plus efficace de leurs ressources pour les organismes de développement économique.

\section{Notes et références}

1.

Laferté, S. (2005). Le soutien aux travailleurs autonomes en exercice : proposition aux organismes de développement économique, thèse de doctorat, Université du Québec à Trois-Rivières.
Lorrain, J. et S. Laferté (2006). «Support Needs of the Young Entrepreneur », Journal of Small Business and Entrepreneurship, vol. 19 no 1, hiver, p. 37-48.

Saint-Pierre, G., S. Laferté et J. Perreault (2003). «The Self-Employed Post-Start-Up Training and Support Needs », ICSB 48th World Conference Proceedings, June 2003, Belfast.

2 Nous désirons ici remercier Emploi-Québec, direction régionale de l'Estrie, le Magazine L'Autonome, le CLD Rimouski Petite Neigette, la SADC Nicolet Bécancour et IDEE - Option Réseau Estrie pour leur collaboration lors de cette enquête.

3 Toute personne intéressée par ces détails peut communiquer avec l'auteure à l'adresse de courriel suivante : laferte.sylvie@teluq.uqam.ca

4 Nous savons bien que plusieurs organismes de développement économique appliquent plusieurs de ces recommandations depuis de nombreuses années. Notre but est de propager l'information et de stimuler la généralisation de pratiques qui pourraient aider les travailleurs autonomes à mieux gérer leur entreprise et d'en assurer, sinon la croissance, du moins une certaine stabilité. 